\title{
A problem with conditions given on inner characteristics and on the line of degeneracy for a mixed-type equation with singular coefficients
}

\author{
Menglibay Kholtojibaevich Ruziev*
}

"Correspondence: mruziev@mail.ru Institute of Mathematics, National University of Uzbekistan named after Mirzo Ulugbek, Tashkent, Uzbekistan

\begin{abstract}
In an unbounded domain, we consider a problem with conditions given on inner characteristics in a hyperbolic part of the considered domain and on some parts of the line of parabolic degeneracy. We prove the unique solvability of the mentioned problem with the help of the extremum principle. The proof of solvability is based on the theory of singular integral equations, Wiener-Hopf equations and Fredholm integral equations.
\end{abstract}

\section{Introduction and formulation of a problem}

Swedish mathematician Gellerstedt [1] investigated a boundary value problem for the equation $y^{m} u_{x x}+u_{y y}=0$ ( $m$ is an odd number), in which the values of a sought function are given on two pieces of characteristics, and on curve $x^{2}+4 /(m+2)^{2} y^{m+2}=1(y>0)$, the value of its derivative is given. This problem has applications in transonic gas dynamics [2].

The Gellerstedt problem and related problems for mixed elliptic-hyperbolic equations were studied in the works [3-5]. The work [6] is devoted to studying the Gellerstedt problem with data on one family of characteristics and with nonlocal gluing conditions. In the work [7] the unique solvability of the Gellerstedt problem for a parabolic-hyperbolic equation of the second kind was studied. The Cauchy problem was investigated by Jachmann and Reissig [8]. Flaisher [9] studied a problem with data on characteristics, outgoing from the origin.

In an unbounded domain, Wolfersdorf [10] investigated the Tricomi problem for the Gellerstedt equation ( $\operatorname{sign} y)|y|^{m} u_{x x}+u_{y y}=0, m>0$.

Boundary value problems for the wave equation and equations of mixed type were investigated in [11]. In the work [12] the general Tricomi-Rassias problem was investigated for the generalized Chaplygin equation. In the paper, the representation of a solution of the general Tricomi-Rassias problem was given for the first time; moreover, the uniqueness and existence of a solution for the problem were proved by a new method. In the works $[13,14]$, fundamental solutions were found and boundary value problems for degenerate elliptic equations were solved.

Due to applications in gas dynamics, the interest in studying boundary value problems for degenerate elliptic and mixed-type equations with singular coefficients has been grow-

\section{Springer}

(c) 2013 Ruziev; licensee Springer. This is an Open Access article distributed under the terms of the Creative Commons Attribution License (http://creativecommons.org/licenses/by/2.0), which permits unrestricted use, distribution, and reproduction in any medium, provided the original work is properly cited. 
ing. Note the latest work [15] on this topic, where the Dirichlet problem for a threedimensional elliptic equation with singular coefficients was investigated. Let $D=D^{+} \cup$ $D^{-} \cup I$ be a domain of the complex plane $z=x+i y$, where $D^{+}$is a half-plane $y>0, D^{-}$ is a finite domain of the half-plane $y<0$, bounded by characteristics $A C$ and $B C$ of the equation

$$
(\operatorname{sign} y)|y|^{m} u_{x x}+u_{y y}+\frac{\alpha_{0}}{|y|^{1-\frac{m}{2}}} u_{x}+\frac{\beta_{0}}{y} u_{y}=0,
$$

outgoing from the points $A(-1,0), B(1,0)$, and by the segment $A B$ of the straight line $y=0$, $I=\{(x, y):-1<x<1, y=0\}$. In equation (1) assume that $m, \alpha_{0}, \beta_{0}$ are some real numbers such that $m>0,-m / 2<\beta_{0}<1,\left|\alpha_{0}\right|<\frac{m+2}{2}$.

Let $D_{R}^{+}$be a finite domain separated from $D^{+}$by the $\operatorname{arc} A_{R} B_{R}$ of the normal curve $x^{2}+$ $4 y^{m+2} /(m+2)^{2}=R^{2},-R \leq x \leq R, 0 \leq y \leq((m+2) R / 2)^{2 /(m+2)}, A_{R}(-R, 0), B_{R}(R, 0)$.

We introduce the following denotations: $\bar{I}_{1}=\{(x, y):-\infty<x \leq-1, y=0\}, \bar{I}_{2}=\{(x, y)$ : $1 \leq x<\infty, y=0\}, C_{0}\left(C_{1}\right)$ are points of intersection of the characteristic $A C(B C)$ with that outgoing from the point $E(c, 0)$, where $c \in I$ is an arbitrary fixed number, $D_{R}=D_{R}^{+} \cup D^{-}$, $D_{R}$ is a subdomain in the unbounded domain $D$.

Consider the diffeomorphism $q(x) \in C^{1}[c, 1]$ mapping the segment $[c, 1]$ into the segment $[-1, c]$; moreover, $q^{\prime}(x)<0, q(c)=c, q(1)=-1$. As an example, we take a linear function $q(x)=p-k x$, where $k=(1+c) /(1-c), p=2 c /(1-c), p-k=-1, p-k c=c$.

Note that in the Gellerstedt problem the values of a sought function in the hyperbolic part of the mixed domain $D$ are given on the characteristics $E C_{0}$ and $E C_{1}:\left.u\right|_{E C_{0}}=\psi_{1}(x)$, $\left.u\right|_{E C_{1}}=\psi_{2}(x)$.

Boundary value problem for equation (1) in the case when $\alpha_{0}=0$, with data on the piece of $A C_{0}$ of the characteristic $A C$ and with inner boundary local shifting condition on $A B$ of the line of degeneracy $y=0$, was studied in the work [16], and with data on pieces $A C_{0}$ and $B C_{1}$ in the work [17].

In the present work, we study a new boundary problem, where characteristic $E C_{0}$ is free from the conditions, and the needed condition of Gellerstedt is replaced by an inner boundary condition with local shifting on the parabolic line of degeneracy.

\section{Formulation of the problem}

Problem $G$. In the domain $D$, find a function $u(x, y)$ satisfying the following conditions:

(1) the function $u(x, y)$ is continuous in any subdomain $\bar{D}_{R}$ of the unbounded domain $D$;

(2) $u(x, y)$ belongs to the space $C^{2}\left(D^{+}\right)$and satisfies equation (1) in this domain;

(3) $u(x, y)$ is a generalized solution from the class $R_{1}\left(\tau^{\prime}(x), v(x) \in H\right)$ in the domain $D^{-}[18]$

(4) the following equalities are fulfilled:

$$
\lim _{R \rightarrow \infty} u(x, y)=0, \quad y \geq 0, \quad R^{2}=x^{2}+4(m+2)^{-2} y^{m+2} ;
$$

(5) $u(x, y)$ satisfies the boundary conditions

$$
\begin{aligned}
& \left.u(x, y)\right|_{y=0}=\tau_{i}(x), \quad \forall x \in \bar{I}_{i}, i=1,2, \\
& \left.u(x, y)\right|_{E C_{1}}=\psi(x), \quad c \leq x \leq(c+1) / 2, \\
& u(q(x), 0)=\mu u(x, 0)+f(x), \quad c \leq x \leq 1,
\end{aligned}
$$


and the conjugation condition

$$
\lim _{y \rightarrow+0} y^{\beta_{0}} u_{y}=\lim _{y \rightarrow-0}(-y)^{\beta_{0}} u_{y}, \quad x \in I \backslash\{c\} .
$$

Moreover, these limits at $x= \pm 1, x=c$ can have singularity of the order less than $1-\alpha-\beta$, where $\alpha=\left(m+2\left(\beta_{0}+\alpha_{0}\right)\right) /(2(m+2)), \beta=\left(m+2\left(\beta_{0}-\alpha_{0}\right)\right) /(2(m+2)), f(x), \psi(x), \tau_{i}(x)$ are given functions such that $f(x) \in C[c, 1] \cap C^{1, \delta_{1}}(c, 1), f(c)=0, f(1)=0, \psi(x) \in C[c,(c+1) / 2] \cap$ $C^{1, \delta_{1}}(c,(c+1) / 2), \psi(c)=0, \mu$-const., the functions $\tau_{i}(x)$ are expressed as $\tau_{i}(x)=\left(1-x^{2}\right) \tilde{\tau}_{i}(x)$ in a neighborhood of the points $x=-1, x=1$, and they satisfy Holder's condition on any intervals $(-N,-1),(1, N), N>1$. For a sufficiently large absolute value $|x|$, they satisfy the inequality $\left|\tau_{i}(x)\right| \leq M|x|^{-\delta}$, where $\delta, M$ are positive constants.

Note that condition (5) is an inner boundary condition with local shifting on the parabolic line of degeneracy [16-18].

\section{The uniqueness of the solution of problem $G$}

Theorem 1 Let conditions $\tau_{i}(x) \equiv 0, i=1,2, \psi(x) \equiv 0, f(x) \equiv 0,0<\mu<1$ be fulfilled. Then problem $G$ has only a trivial solution.

Proof Solution of the modified Cauchy problem for equation (1) in the domain $D^{-}$, satisfying initial conditions $\lim _{y \rightarrow-0} u(x, y)=\tau(x), x \in \bar{I}, \lim _{y \rightarrow-0}(-y)^{\beta_{0}} u_{y}=v(x), x \in I$, is given by the formula [18]

$$
\begin{aligned}
u(x, y)= & \gamma_{1} \int_{-1}^{1} \tau\left[x+\frac{2 t}{m+2}(-y)^{(m+2) / 2}\right](1+t)^{\beta-1}(1-t)^{\alpha-1} d t \\
& +\gamma_{2}(-y)^{1-\beta_{0}} \int_{-1}^{1} v\left[x+\frac{2 t}{m+2}(-y)^{(m+2) / 2}\right](1+t)^{-\alpha}(1-t)^{-\beta} d t
\end{aligned}
$$

where $\gamma_{1}=\Gamma(\alpha+\beta) 2^{1-\alpha-\beta} / \Gamma(\beta) \Gamma(\alpha), \gamma_{2}=-\Gamma(2-\alpha-\beta) 2^{\alpha+\beta-1} /\left(\left(1-\beta_{0}\right) \Gamma(1-\alpha) \Gamma(1-\beta)\right)$, $\Gamma(z)$ is Euler's gamma function [19].

Satisfying (7) to condition (4), after some evaluations, we obtain

$$
v(X)=\gamma D_{c, X}^{1-\alpha-\beta} \tau(X)+\Psi(X), \quad X \in(c, 1),
$$

where

$$
\Psi(X)=\frac{(X-c)^{\alpha} D_{c, X}^{1-\beta} \psi((X+c) / 2)}{\gamma_{2}\left(\frac{m+2}{2}\right)^{1-\alpha-\beta} \Gamma(1-\beta)}, \quad X=2 x-c,
$$

$\gamma=2 \Gamma(1-\alpha) \Gamma(\alpha+\beta)((m+2) / 4)^{\alpha+\beta} /(\Gamma(\beta) \Gamma(1-\alpha-\beta)), D_{c, x}^{l}$ is a differential operator of fractional order in a sense of Riemann-Liouville [19].

Equality (8) is the first functional relation between functions $\tau(x)$ and $v(x)$, on an interval of the axis $y=0$ reduced from the domain $D^{-}$.

Prove that if $\tau_{i}(x) \equiv 0, i=1,2, \psi(x) \equiv 0, f(x) \equiv 0,0<\mu<1$, then the solution of problem $G$ in the domain $D^{+} \cup I_{1} \cup \bar{I} \cup I_{2}$ is trivial by virtue of equality (2).

Let $\left(x_{0}, y_{0}\right)$ be a point of positive maximum of the function $u(x, y)$ in the domain $\bar{D}_{R}^{+}$. By virtue of (2) for $\forall \varepsilon>0$, there exists $R_{0}=R_{0}(\varepsilon)$ such that at $R>R_{0}(\varepsilon)$

$$
|u(x, y)|<\varepsilon, \quad(x, y) \in A_{R} B_{R} .
$$


Considering designation $u(x, 0)=\tau(x), x \in \bar{I}$, condition (5) can be written as

$$
\tau(q(x))=\mu \tau(x)+f(x), \quad x \in[c, 1] .
$$

Hence, at $x=c$ (where $f(x) \equiv 0$ ) we get $\tau(q(c))=\mu \tau(c)$. Then, taking $q(c)=c$ into account, we have $\tau(c)(1-\mu)=0$, i.e., $\tau(c)=0$. By the Hopf principle [20], the function $u(x, y)$ cannot reach its positive maximum and negative minimum on inner points of the domain $\bar{D}_{R}^{+}$. By virtue of $0<\mu<1$, from (10) (where $f(x) \equiv 0$ ) it follows that there are no points of extremum in the interval $(-1, c)$ of the axis $y=0$.

Assume that the sought function reaches its positive maximum and negative minimum on points of the interval $(c, 1)$ of the axis $y=0$.

Let $\left(x_{0}, 0\right)$ (where $\left.x_{0} \in(c, 1)\right)$ be a point of positive maximum (negative minimum) of the function $u(x, 0)=\tau(x)$. Then [21]

$$
v\left(x_{0}\right)<0 \quad\left(v\left(x_{0}\right)>0\right) .
$$

It is well known that on the point of positive maximum (negative minimum) of the function $\tau(x)$ for the differential operators of fractional order, the following inequality $D_{c, x_{0}}^{1-\alpha-\beta} \tau(x)>0\left(D_{c, x_{0}}^{1-\alpha-\beta} \tau(x)<0\right)$ holds. Hence, considering (8) (where $\left.\Psi(X) \equiv 0\right)$, we deduce

$$
v\left(x_{0}\right)=\gamma D_{c, x_{0}}^{1-\alpha-\beta} \tau(x)>0 \quad\left(v\left(x_{0}\right)=\gamma D_{c, x_{0}}^{1-\alpha-\beta} \tau(x)<0\right) .
$$

Inequalities (11) and (12) contradict the gluing condition (6), therefore $x_{0} \notin(c, 1)$. Hence, there is no point of positive maximum (negative minimum) of the function $u(x, y)$ in the interval $A B$. Let $R>R_{0}$. By the Hopf principle and the statements obtained above, it follows that $\left(x_{0}, y_{0}\right) \in A_{R} B_{R}$, and by virtue of (9), $\left|u\left(x_{0}, y_{0}\right)\right|<\varepsilon$. Consequently, $|u(x, y)|<\varepsilon$ for $\forall(x, y) \in \bar{D}_{R}^{+}$. From here, due to arbitrariness of $\varepsilon$ at $R \rightarrow \infty$, we conclude that $u(x, y) \equiv 0$ in the domain $D^{+} \cup I_{1} \cup \bar{I} \cup I_{2}$. Then

$$
\lim _{y \rightarrow+0} u(x, y)=0, \quad x \in \bar{I}, \quad \lim _{y \rightarrow+0} y^{\beta_{0}} u_{y}=0, \quad x \in I .
$$

Considering (13), due to continuity of the solution in $\bar{D}_{R}^{+}$and conjugation condition (6), we restore the desired function $u(x, y)$ in the domain $D^{-}$as a solution of the modified Cauchy problem with homogeneous data and get $u(x, y) \equiv 0$ in the domain $\bar{D}^{-}$. The proof of Theorem 1 is complete.

\section{The existence of the solution of problem $G$}

Theorem 2 Let the following conditions be fulfilled: $q(x)=p-k x, 0<\mu<1, \lambda \mu k^{\alpha+\beta+\theta-\frac{1}{2}} \times$ $\pi e^{2 b \pi}<1, \frac{1}{2}-(\alpha+\beta+\theta)<0, \lambda=\frac{\gamma \Gamma(1-\alpha-\beta)}{\frac{e^{-b \pi}}{k_{2}\left(1-\beta_{0}\right)(m+2) / 2}+\frac{\pi}{\sin (\alpha+\beta) \pi}\left(e^{b \pi}-e^{-b \pi} \cos (\alpha+\beta) \pi\right)}>0$, where $b=-\frac{\alpha_{0}}{m+2}$, $\theta=\frac{\operatorname{arctg}(\lambda \pi)}{\pi}, k_{2}=\frac{1}{4 \pi}\left(\frac{4}{m+2}\right)^{2-2 a} \frac{\Gamma(1-l) \Gamma(1-\bar{l})}{\Gamma(2-l-\bar{l})}, 2 a=\alpha+\beta, l=a+b i$. Then problem $G$ has a solution.

Proof The solution of the Dirichlet problem satisfying condition (3) and the requirement $u(x, 0)=\tau(x), x \in \bar{I}$, can be represented in the form

$$
u(x, y)=k_{2}\left(1-\beta_{0}\right) y^{1-\beta_{0}} \int_{-1}^{1} \tau(t)\left(r_{0}^{2}\right)^{a-1} \exp \left(-2 b \arcsin \frac{t-x}{r_{0}}\right) d t+F_{1}(x, y) \text {, }
$$


where $r_{0}^{2}=(x-t)^{2}+\frac{4}{(m+2)^{2}} y^{m+2}$

$$
\begin{aligned}
F_{1}(x, y)= & k_{2}\left(1-\beta_{0}\right) y^{1-\beta_{0}}\left(\int_{-\infty}^{-1} \tau_{1}(t)\left(r_{0}^{2}\right)^{a-1} \exp \left(-2 b \arcsin \frac{t-x}{r_{0}}\right) d t\right. \\
& \left.+\int_{1}^{\infty} \tau_{2}(t)\left(r_{0}^{2}\right)^{a-1} \exp \left(-2 b \arcsin \frac{t-x}{r_{0}}\right) d t\right) .
\end{aligned}
$$

Differentiating (14) along $y$ and considering the equality

$$
\begin{aligned}
& \frac{\partial}{\partial y}\left\{y^{1-\beta_{0}}\left(r_{0}^{2}\right)^{a-1} \exp \left(-2 b \arcsin \frac{t-x}{r_{0}}\right)\right\} \\
& \quad=\frac{m+2}{2} y^{-\beta_{0}} \frac{\partial}{\partial t}\left\{(x-t)\left(r_{0}^{2}\right)^{a-1} \exp \left(-2 b \arcsin \frac{t-x}{r_{0}}\right)\right\},
\end{aligned}
$$

we get

$$
\begin{aligned}
\frac{\partial u}{\partial y}= & k_{2}\left(1-\beta_{0}\right) \frac{m+2}{2} y^{-\beta_{0}} \int_{-1}^{1} \tau(t) \frac{\partial}{\partial t}\left[(x-t)\left(r_{0}^{2}\right)^{a-1} \exp \left(-2 b \arcsin \frac{t-x}{r_{0}}\right)\right] d t \\
& +\frac{\partial F_{1}(x, y)}{\partial y} .
\end{aligned}
$$

Integrating by parts (taking $\tau(-1)=0, \tau(1)=0$ into account), after some evaluations, we have

$$
\begin{aligned}
\frac{\partial u}{\partial y}= & -k_{2}\left(1-\beta_{0}\right) \frac{m+2}{2} y^{-\beta_{0}} \int_{-1}^{1} \tau^{\prime}(t)\left[(x-t)\left(r_{0}^{2}\right)^{a-1} \exp \left(-2 b \arcsin \frac{t-x}{r_{0}}\right)\right] d t \\
& +\frac{\partial F_{1}(x, y)}{\partial y} .
\end{aligned}
$$

Multiplying both sides of (15) to $y^{\beta_{0}}$, and passing to the limit at $y \rightarrow+0$, we obtain

$$
\begin{aligned}
& v(x)=-k_{2}\left(1-\beta_{0}\right) \frac{m+2}{2} \int_{-1}^{1} \tau^{\prime}(t)(x-t)|x-t|^{2 a-2} \exp \left(-2 b \arcsin \frac{t-x}{|t-x|}\right) d t+\Phi(x) \\
& x \in(-1,1)
\end{aligned}
$$

where $\Phi(x)=\lim _{y \rightarrow+0} y^{\beta_{0}} \frac{\partial F_{1}(x, y)}{\partial y}=k_{2}\left(1-\beta_{0}\right)^{2}\left(e^{b \pi} \int_{-\infty}^{-1} \frac{\tau_{1}(t) d t}{(x-t)^{2-2 a}}+e^{-b \pi} \int_{1}^{\infty} \frac{\tau_{2}(t) d t}{(t-x)^{2-2 a}}\right)$.

Equation (16) is the second functional relation between unknown functions $v(x), \tau(x)$ in the interval $I$ of the axis $y=0$ deduced from the upper half-plane.

Note that relation $(16)$ is valid for the whole $I$. Breaking $(-1,1)$ into the intervals $(-1, c)$ and $(c, 1)$, then into the integral with bounds $(-1, c)$, we make change of variables $t=q(s)=$ $p-k s$. By virtue of (10), from (16) we obtain

$$
\begin{aligned}
v(x)= & -k_{2}\left(1-\beta_{0}\right) \frac{m+2}{2} \\
& \times\left[-\mu e^{b \pi} \int_{c}^{1} \frac{\tau^{\prime}(s) d s}{(x-q(s))^{1-2 a}}-e^{b \pi} \int_{c}^{1} \frac{f^{\prime}(s) d s}{(x-q(s))^{1-2 a}}+e^{b \pi} \int_{c}^{x} \tau^{\prime}(t)(x-t)^{2 a-1} d t\right. \\
& \left.-e^{-b \pi} \int_{x}^{1} \tau^{\prime}(t)(t-x)^{2 a-1} d t\right]+\Phi(x), \quad x \in(c, 1) .
\end{aligned}
$$


Considering (6), excluding the function $v(x)$ from (8) and (17), we deduce

$$
\begin{aligned}
& \frac{-2 \gamma}{k_{2}\left(1-\beta_{0}\right)(m+2)} D_{c, x}^{1-\alpha-\beta} \tau(x)+F_{0}(x) \\
& \quad=-\mu e^{b \pi} \int_{c}^{1} \frac{\tau^{\prime}(s) d s}{(x-q(s))^{1-2 a}}+e^{b \pi} \int_{c}^{x} \frac{\tau^{\prime}(t) d t}{(x-t)^{1-2 a}}-e^{-b \pi} \int_{x}^{1} \frac{\tau^{\prime}(t) d t}{(t-x)^{1-2 a}}, \\
& F_{0}(x)=-\frac{2(\Psi(x)-\Phi(x))}{k_{2}\left(1-\beta_{0}\right)(m+2)}+e^{b \pi} \int_{c}^{1} \frac{f^{\prime}(s) d s}{(x-q(s))^{1-2 a}} .
\end{aligned}
$$

Applying the operator $\Gamma(1-\alpha-\beta) D_{c, x}^{\alpha+\beta-1}$ to the both sides of (18), considering $D_{c, x}^{\alpha+\beta-1} \times$ $D_{c, x}^{1-\alpha-\beta} \tau(x)=\tau(x)$, we have

$$
\begin{aligned}
& \frac{-2 \gamma}{k_{2}\left(1-\beta_{0}\right)(m+2)} \Gamma(1-\alpha-\beta) \tau(x)+\Gamma(1-\alpha-\beta) D_{c, x}^{\alpha+\beta-1} F_{0}(x) \\
& =\Gamma(1-\alpha-\beta) D_{c, x}^{\alpha+\beta-1} \\
& \quad \times\left[-\mu e^{b \pi} \int_{c}^{1} \frac{\tau^{\prime}(s) d s}{(x-q(s))^{1-2 a}}+e^{b \pi} \int_{c}^{x} \frac{\tau^{\prime}(t) d t}{(x-t)^{1-2 a}}-e^{-b \pi} \int_{x}^{1} \frac{\tau^{\prime}(t) d t}{(t-x)^{1-2 a}}\right], \\
& x \in[c, 1] .
\end{aligned}
$$

Further, one can easily prove that

$$
\begin{aligned}
& -\mu e^{b \pi} \Gamma(1-\alpha-\beta) D_{c, x}^{\alpha+\beta-1} \int_{c}^{1} \frac{\tau^{\prime}(s) d s}{(x-q(s))^{1-\alpha-\beta}} \\
& =\mu e^{b \pi} \int_{c}^{1}\left(\frac{x-c}{c-q(s)}\right)^{1-\alpha-\beta} \frac{\tau(s) q^{\prime}(s) d s}{x-q(s)} \\
& e^{b \pi} \Gamma(1-\alpha-\beta) D_{c, x}^{\alpha+\beta-1} \int_{c}^{x} \frac{\tau^{\prime}(t) d t}{(x-t)^{1-\alpha-\beta}}=e^{b \pi} \Gamma(\alpha+\beta) \Gamma(1-\alpha-\beta) \tau(x), \\
& e^{-b \pi} \Gamma(1-\alpha-\beta) D_{c, x}^{\alpha+\beta-1} \int_{x}^{1} \frac{\tau^{\prime}(t) d t}{(t-x)^{1-\alpha-\beta}} \\
& =e^{-b \pi} \pi \operatorname{ctg}((\alpha+\beta) \pi) \tau(x)+e^{-b \pi} \int_{c}^{1}\left(\frac{t-c}{x-c}\right)^{\alpha+\beta-1} \frac{\tau(t) d t}{t-x} .
\end{aligned}
$$

Substituting (20)-(22) into (19), after some calculations, we get the singular integral equation regarding the function $\tau(x)$ :

$$
\begin{aligned}
\tau(x) & -\lambda \int_{c}^{1}\left(\frac{x-c}{t-c}\right)^{1-\alpha-\beta} \frac{\tau(t) d t}{t-x} \\
& =-\mu \lambda e^{2 b \pi} \int_{c}^{1}\left(\frac{x-c}{c-q(s)}\right)^{1-\alpha-\beta} \frac{\tau(s) q^{\prime}(s) d s}{x-q(s)}+F(x),
\end{aligned}
$$

where $F(x)=\Gamma(1-\alpha-\beta) \lambda e^{b \pi} D_{c, x}^{\alpha+\beta-1} F_{0}(x), F(x) \in C[c, 1] \cap C^{0, \bar{\gamma}}(c, 1), \bar{\gamma}>1-\beta$. The integral operator on the right-hand side of (23) is not regular since expression under the integral has isolated singularity of the first order at $x=c, s=c$, and this is why this item is written separately. Setting the right-hand side of (23) temporarily as a known function, we rewrite 
it as follows:

$$
\tau(x)-\lambda \int_{c}^{1}\left(\frac{x-c}{t-c}\right)^{1-\alpha-\beta} \frac{\tau(t) d t}{t-x}=g_{0}(x),
$$

where

$$
g_{0}(x)=-\mu \lambda e^{2 b \pi} \int_{c}^{1}\left(\frac{x-c}{c-q(s)}\right)^{1-\alpha-\beta} \frac{\tau(s) q^{\prime}(s) d s}{x-q(s)}+F(x) .
$$

Setting $(x-c)^{\alpha+\beta-1} \tau(x)=\rho(x),(x-c)^{\alpha+\beta-1} g_{0}(x)=g(x)$, we rewrite equation (24) as

$$
\rho(x)-\lambda \int_{c}^{1} \frac{\rho(t) d t}{t-x}=g(x) \text {. }
$$

We search for a solution of equation (26) in the class of functions, satisfying Holder's condition on $(c, 1)$ and bounded at $x \rightarrow 1$, and at $x \rightarrow c$ they can tend to infinity with order less than $1-\alpha-\beta$. Index $\chi$ of this class is equal to zero, and solution can be explicitly found by the method of Carleman-Vekua [22]:

$$
\rho(x)=\frac{g(x)}{1+\lambda^{2} \pi^{2}}+\frac{\lambda}{1+\lambda^{2} \pi^{2}}\left(\frac{1-x}{x-c}\right)^{\theta} \int_{c}^{1}\left(\frac{t-c}{1-t}\right)^{\theta} \frac{g(t) d t}{t-x},
$$

where $\theta=\psi / \pi, \psi=\operatorname{arctg}(\lambda \pi), 0<\theta<1 / 2$. From here, according to designation, we have

$$
\tau(x)=\frac{g_{0}(x)}{1+\lambda^{2} \pi^{2}}+\frac{\lambda}{1+\lambda^{2} \pi^{2}}\left(\frac{1-x}{x-c}\right)^{\theta} \int_{c}^{1}\left(\frac{t-c}{1-t}\right)^{\theta}\left(\frac{t-c}{1-t}\right)^{\alpha+\beta-1} \frac{g_{0}(t) d t}{t-x} .
$$

Substituting (25) into (27), after some evaluations, we obtain

$$
\begin{aligned}
\tau(x)= & \frac{-\lambda \mu e^{2 b \pi}}{1+\lambda^{2} \pi^{2}} \int_{c}^{1}\left(\frac{x-c}{c-q(s)}\right)^{1-\alpha-\beta} \frac{\tau(s) q^{\prime}(s) d s}{x-q(s)} \\
& -\frac{\lambda^{2} \mu e^{2 b \pi}}{1+\lambda^{2} \pi^{2}}\left(\frac{1-x}{x-c}\right)^{\theta} \int_{c}^{1}\left(\frac{x-c}{c-q(s)}\right)^{1-\alpha-\beta} \tau(s) q^{\prime}(s) d s \\
& \times \int_{c}^{1}\left(\frac{t-c}{1-t}\right)^{\theta} \frac{d t}{(t-q(s))(t-x)}+F_{1}(x),
\end{aligned}
$$

where

$$
F_{1}(x)=\frac{F(x)}{1+\lambda^{2} \pi^{2}}+\frac{\lambda}{1+\lambda^{2} \pi^{2}}\left(\frac{1-x}{x-c}\right)^{\theta} \int_{c}^{1}\left(\frac{t-c}{1-t}\right)^{\theta}\left(\frac{t-c}{x-c}\right)^{\alpha+\beta-1} \frac{F(t) d t}{t-x} .
$$

Equation (28), by virtue of $q(x)=p-k x$, where $k=(1+c) /(1-c), p=2 c /(1-c)$, can be rewritten as

$$
\begin{aligned}
\tau(x)= & \frac{-\lambda \mu k^{\alpha+\beta} e^{2 b \pi}}{1+\lambda^{2} \pi^{2}} \int_{c}^{1}\left(\frac{x-c}{s-c}\right)^{1-\alpha-\beta} \frac{\tau(s) d s}{(p-k s)-x} \\
& +\frac{\lambda^{2} \mu k^{\alpha+\beta} e^{2 b \pi}}{1+\lambda^{2} \pi^{2}}\left(\frac{1-x}{x-c}\right)^{\theta} \int_{c}^{1}\left(\frac{x-c}{s-c}\right)^{1-\alpha-\beta} \tau(s) d s \\
& \times \int_{c}^{1}\left(\frac{t-c}{1-t}\right)^{\theta} \frac{d t}{(t-x)(t-(p-k s))}+F_{1}(x), \quad x \in[c, 1] .
\end{aligned}
$$


Further, in (29) we calculate the inner integral

$$
A(x, s)=\int_{c}^{1}\left(\frac{t-c}{1-t}\right)^{\theta} \frac{d t}{(t-x)(t+k s-p)}
$$

Expanding the rational factor of the integrand in simple fractions and completing simple calculations, we have

$$
A(x, s)=\frac{1}{x+k s-p}\left(-\pi \operatorname{ctg}(\pi \theta)(1-x)^{-\theta}(x-c)^{\theta}+\frac{\pi}{\sin (\pi \theta)} k^{\theta}\left(\frac{s-c}{1+k s-p}\right)^{\theta}\right) .
$$

Substituting (30) into (29), considering $\operatorname{ctg}(\operatorname{arctg} x)=\frac{1}{x}, x \neq 0$, after some calculations, we get

$$
\begin{aligned}
\tau(x)= & -\frac{\lambda^{2} \mu k^{\alpha+\beta+\theta} e^{2 b \pi}}{1+\lambda^{2} \pi^{2}} \frac{\pi}{\sin (\pi \theta)} \\
& \times \int_{c}^{1}\left(\frac{1-x}{1+k s-p}\right)^{\theta}\left(\frac{x-c}{s-c}\right)^{1-\alpha-\beta-\theta} \frac{\tau(s) d s}{(p-k s)-x}+F_{1}(x), \quad x \in[c, 1] .
\end{aligned}
$$

Considering formulas $\sin (\operatorname{arctg} x)=\frac{x}{\sqrt{1+x^{2}}}, \frac{\pi}{\sin (\pi \theta)}=\frac{\sqrt{1+\lambda^{2} \pi^{2}}}{\lambda}$, we rewrite equality (31) as

$$
\begin{aligned}
\tau(x)= & -\frac{\lambda \mu k^{\alpha+\beta+\theta} e^{2 b \pi}}{\sqrt{1+\lambda^{2} \pi^{2}}} \\
& \times \int_{c}^{1}\left(\frac{1-x}{1+k s-p}\right)^{\theta}\left(\frac{x-c}{s-c}\right)^{1-\alpha-\beta-\theta} \frac{\tau(s) d s}{(p-k s)-x}+F_{1}(x), \quad x \in[c, 1] .
\end{aligned}
$$

In (32) allocating a characteristic part, we get

$$
\begin{aligned}
\tau(x)= & -\frac{\lambda \mu k^{\alpha+\beta+\theta} e^{2 b \pi}}{\sqrt{1+\lambda^{2} \pi^{2}}} \\
& \times \int_{c}^{1}\left(\frac{x-c}{s-c}\right)^{1-\alpha-\beta-\theta} \frac{\tau(s) d s}{(p-k s)-x}+R_{1}[\tau(x)]+F_{1}(x), \quad x \in[c, 1],
\end{aligned}
$$

where

$$
\begin{aligned}
R_{1}[\tau(x)]= & \frac{-\lambda \mu k^{\alpha+\beta+\theta} e^{2 b \pi}}{\sqrt{1+\lambda^{2} \pi^{2}}} \\
& \times \int_{c}^{1}\left(\frac{x-c}{s-c}\right)^{1-\alpha-\beta-\theta} \frac{\tau(s) d s}{(p-k s)-x}\left[\left(\frac{1-x}{1-(p-k s)}\right)^{\theta}-1\right] d s
\end{aligned}
$$

is a regular operator. We rewrite equation (33) as

$$
\begin{aligned}
\tau(x)= & \frac{\lambda \mu k^{\alpha+\beta+\theta} e^{2 b \pi}}{\sqrt{1+\lambda^{2} \pi^{2}}} \\
& \times \int_{c}^{1}\left(\frac{x-c}{s-c}\right)^{1-\alpha-\beta-\theta} \frac{\tau(s) d s}{(s-c)\left(k+\frac{x-c}{s-c}\right)}+R_{1}[\tau(x)]+F_{1}(x), \quad x \in[c, 1] .
\end{aligned}
$$


Making change of variables $x=c+(1-c) e^{-\xi}, s=c+(1-c) e^{-t}$ and designating $\rho(\xi)=$ $\tau\left(c+(1-c) e^{-\xi}\right) e^{\left(\frac{1}{2}-(\alpha+\beta+\theta)\right) \xi}$, we write equation (34) as

$$
\rho(\xi)=\frac{\lambda \mu k^{\alpha+\beta+\theta} e^{2 b \pi}}{\sqrt{1+\lambda^{2} \pi^{2}}} \int_{0}^{\infty} \frac{\rho(t) d t}{k e^{\frac{\xi-t}{2}}+e^{-\frac{\xi-t}{2}}}+R_{2}[\rho(\xi)]+F_{2}(\xi), \quad \xi \in[0, \infty)
$$

where

$$
\begin{aligned}
& R_{2}[\rho(\xi)]=R_{1}\left[\tau\left(c+(1-c) e^{-\xi}\right)\right] e^{\left(\frac{1}{2}-(\alpha+\beta+\theta)\right) \xi}, \\
& F_{2}(\xi)=F_{1}\left(c+(1-c) e^{-\xi}\right) e^{\left(\frac{1}{2}-(\alpha+\beta+\theta)\right) \xi} .
\end{aligned}
$$

We introduce the following notations:

$$
H(\xi)=\frac{\lambda \mu k^{\alpha+\beta+\theta} e^{2 b \pi}}{\sqrt{1+\lambda^{2} \pi^{2}}} \frac{1}{k e^{\xi / 2}+e^{-\xi / 2}} .
$$

By virtue of (36), equation (35) has the form

$$
\rho(\xi)=\int_{0}^{\infty} H(\xi-t) \rho(t) d t+R_{2}[\rho(\xi)]+F_{2}(\xi), \quad \xi \in[0, \infty) .
$$

Equation (37) is an integral equation of Wiener-Hopf [23]. Functions $H(\xi), F_{2}(\xi)$ are indicative of decrease at infinity; moreover, $H^{\prime}(\xi) \in C(0, \infty), F_{2}(\xi) \in H^{\alpha_{1}}(0, \infty)$. Consequently, $H_{2}(\xi), F_{2}(\xi) \in L_{2} \cap H^{\alpha_{1}}$, and a solution of equation (37) will be sought in the class $\{0\}$ [23]. Using Fourier transformation, equation (37) is deduced to the Riemann problem and is solved in quadratures. Fredholm's theorems are only valid in one particular case, when the index of these equations is equal to zero.

Calculate the index of the expression $1-H^{\wedge}(\xi)$, where

$$
H^{\wedge}(\xi)=\int_{-\infty}^{\infty} e^{i \xi t} H(t) d t=\frac{\lambda \mu k^{\alpha+\beta+\theta} e^{2 b \pi}}{\sqrt{1+\lambda^{2} \pi^{2}}} \int_{-\infty}^{\infty} \frac{e^{i \xi t} d t}{k e^{t / 2}+e^{-t / 2}} .
$$

Using the theory of residues, we find

$$
\int_{-\infty}^{\infty} \frac{e^{i \xi t} d t}{k e^{t / 2}+e^{-t / 2}}=\frac{\pi e^{-i \xi \ln k}}{\sqrt{k} \operatorname{ch}(\pi \xi)}
$$

Substituting (39) into (38), we have

$$
H^{\wedge}(\xi)=\frac{\lambda \mu k^{\alpha+\beta+\theta-\frac{1}{2}} \pi e^{2 b \pi}}{\sqrt{1+\lambda^{2} \pi^{2}}} \frac{e^{-i \xi \ln k}}{\operatorname{ch}(\pi \xi)} .
$$

By virtue of the condition $\lambda \mu k^{\alpha+\beta+\theta-\frac{1}{2}} \pi e^{2 b \pi}<1$, and since

$$
\begin{aligned}
\operatorname{Re}\left(H^{\wedge}(\xi)\right) & =\operatorname{Re}\left(\frac{\lambda \mu k^{\alpha+\beta+\theta-\frac{1}{2}} \pi e^{2 b \pi}}{\sqrt{1+\lambda^{2} \pi^{2}}} \frac{e^{-i \xi \ln k}}{\operatorname{ch}(\pi \xi)}\right) \\
& =\frac{\lambda \mu k^{\alpha+\beta+\theta-\frac{1}{2}} \pi e^{2 b \pi}}{\sqrt{1+\lambda^{2} \pi^{2}}} \frac{1}{\operatorname{ch}(\pi \xi)} \cos (\xi \ln k)<\lambda \mu k^{\alpha+\beta+\theta-\frac{1}{2}} \pi e^{2 b \pi}<1,
\end{aligned}
$$


then $\operatorname{Re}\left(1-H^{\wedge}(\xi)\right)>0$. Therefore, the index of equation $(37) \chi=-\operatorname{Ind}\left(1-H^{\wedge}(\xi)\right)=0$, i.e., the variation of the argument of the expression $1-H^{\wedge}(\xi)$ on the real axis expressed in complete revolutions equals zero [23]. Hence, taking into account the fact that the solution to problem $G$ is unique, we deduce the unique solvability of equation (37) and, consequently, that of problem $G$. Theorem 2 is proved.

\section{Competing interests}

The author declares that he has no competing interests.

\section{Received: 11 March 2013 Accepted: 12 August 2013 Published: 12 September 2013}

\section{References}

1. Gellerstedt, S: Quelques problèmes mixtes pour l'équation $y^{m} z_{x x}+z_{y y}=0$. Arkiv f. M.A.O.F. Bd. 26A 3, 1-32 (1938)

2. Bers, L: Mathematical Aspects of Subsonic and Transonic Gas Dynamics. Wiley, New York (1958)

3. Krikunov, YM: On one Gellerstedt problem. Izv. Vysš. Učebn. Zaved., Mat. 6, 56-59 (1975)

4. Solodovnikov, YM: Generalized Gellerstedt problem for a particular case of the equation $K(y) u_{x x}+u_{y y}=0$. Tr. Sem. Po Kr. Zad. Kazan, Vyp. 10, 130-139 (1973)

5. Khairullin, RS: On the Gellerstedt problem for the second kind equation. Izv. Vysš. Učebn. Zaved., Mat. 10, 72-77 (2005)

6. Mirsaburov, M, Eshonkulov, BO: The Gellerstedt problem with data on characteristics of one family and with nonlocal gluing conditions. Izv. KBNTs. RAN 1(18), 48-53 (2002)

7. Mamadaliev, NK: The Gellerstedt problem for a parabolic-hyperbolic equation of the second kind. Int. J. Dyn. Syst. Differ. Equ. 1(2), 102-108 (2007)

8. Jachmann, K, Reissig, M: Cauchy problem for linear thermo-elastic systems. Part 1: A unified approach. Int. J. Dyn. Syst. Differ. Equ. 2(3/4), 170-201 (2009)

9. Flaisher, NM: Some problems with data on characteristics for mixed-type equations. Rev. Roum. Math. Pures Appl. 12(8), 1053-1058 (1967)

10. Wolfersdorf, L: Abelshe Integralgleichungen und Randwertprobleme fur die verallgemeinerte Tricomi-Gleichung. Math. Nachr. 29(H3/4), 161-178 (1965)

11. Protter, MH: New boundary value problems for the wave equation and equations of mixed type. J. Ration. Mech. Anal. 3(4), 435-446 (1954)

12. Wen, G, Chen, D, Cheng, X: General Tricomi-Rassias problem and oblique derivative problem for generalized Chaplygin equations. J. Math. Anal. Appl. 333, 679-694 (2007)

13. Hasanov, A, Rassias, JM: Fundamental solutions of two degenerated elliptic equations and solutions of boundary value problems in infinite area. Int. J. Appl. Math. Stat. 8(M07), 87-95 (2007)

14. Hasanov, A, Rassias, JM, Turaev, M: Fundamental solution for the generalized elliptic Gellerstedt equation. In: Functional Equations, Difference Inequalities and Ulam Stability Notions, No. 6, pp. 73-83. Nova Science Publishers, New York (2010)

15. Karimov, ET, Nieto, JJ: The Dirichlet problem for a 3D elliptic equation with two singular coefficients. Comput. Math Appl. 62(1), 214-224 (2011)

16. Mirsaburov, M, Ruziev, MK: A boundary value problem for a class of mixed-type equations in an unbounded domain. Differ. Equ. 47(1), 112-119 (2011)

17. Ruziev, MK: Problems with shifts for mixed elliptic-hyperbolic equations. Russ. Math. 56(1), 66-75 (2012)

18. Salakhitdinov, MS, Mirsaburov, M: Nonlocal Problems for Equations of Mixed Type with Singular Coefficients. Universitet, Tashkent (2005)

19. Smirnov, MM: Equations of Mixed Type. Vyssh. Shkola, Moscow (1985)

20. Bitsadze, AV: Some Classes of Partial Differential Equations. Nauka, Moscow (1981)

21. Ruziev, MK: A boundary value problems for equation of mixed type with singular coefficients in an unbounded domains. Diss., Tashkent (1999)

22. Muskhelishvili, NI: Singular Integral Equations. Boundary-Value Problems of Function Theory and Some of Their Applications to Mathematical Physics. Nauka, Moscow (1968)

23. Gakhov, FD, Cherskii, Yl: Convolution Type Equations. Nauka, Moscow (1978) 